\title{
Principles of Diagnosis
}

When starting, the simplest approach is to examine the epidermis, then the dermis and finally the subcutaneous fat. This approach reduces the likelihood of overlooking something. For this reason, we have arranged this book, starting with epidermal changes and moving down through dermal and subcutaneous lesions.

Inflammatory dermatoses are best approached by identifying first the pattern of inflammation (superficial vs. superficial and deep vs. subcutaneous; with and without epidermal involvement) and studying the cellular composition (lymphocytes, macrophages, neutrophils, eosinophils, and mast cells). Cutaneous tumors are approached just like all other tumors considering the pattern (symmetry, circumscription, level of invasion) and cytomorphology (nuclear pleomorphism and mitotic activity). Increasingly the final answer is based on the expression of a pattern of specific tumor antigens (immunohistochemical profile).

Clinicopathologic correlation: This is the crux and beauty of dermatopathology; no other branch of medicine has so many different names for the appearance of an organ and in many cases, each of the peculiar names has a histological correlate. On the other hand, the skin can only react in so many ways, so that some patterns such as "superficial lymphocytic perivascular infiltrate with sparse admixture of eosinophils" can be associated with many different diagnoses ranging from viral exanthem to drug reaction or bullous pemphigoid. The digital age has made it convenient to provide the dermatopathologist not only with a detailed clinical history but also with clinical pictures (e.g., digital images), both of which increase the chances of the clinician receiving a helpful diagnosis. 